\title{
A 3D object detection framework for self-driving using YOLOv4
}

\author{
Zhen $\mathrm{Li}^{\mathrm{a}, *}$, Yuren Du ${ }^{\mathrm{b}}$, Qingqing Hong ${ }^{\mathrm{b}}$, Seiichi Serikawa ${ }^{\mathrm{a}}$, Lifeng Zhang ${ }^{\mathrm{a}}$ \\ ${ }^{a}$ Kyushu Institute of Technology, Kitakyushu, Japan \\ b Yangzhou University, Yangzhou, China \\ *Corresponding Author: li.zhen799@ mail.kyutech.jp
}

\begin{abstract}
As the continuous evolution of the traditional vehicles' industry, the self-driving technology has become an indispensable part of the intelligent vehicles in contemporary age. Object detection is the most core technique in self-driving to provide great perception abilities for smart cars. 3D object detector could acquire more effective metric information for self-driving in 3D spatial coordination system. While most of the 3D detection frameworks based on 2D detectors actually did a 2D detection and transformed the 2D bounding boxes into 3D bounding boxes, neglecting the regression of metric information in vertical coordination axis. So this paper focused on a real 3D object detection framework using 2D based neural network YOLOv4, ${ }^{\text {(II) }}$ in regard of the realtime performance. The proposed framework transformed YOLOv4 neural network into a 3D object detector, and it can detect 3 classifications of objects in self-driving application. The proposed $3 \mathrm{D}$ object detection framework is proved to achieved a state-of-the-art accurate performance in real-time self-driving applications. The average precision of 3D detection could achieve $65.86 \%$ at the real-time performance of 27.6 FPS.
\end{abstract}

keywords: 3D object detection, YOLOv4, Self-driving, Real-time.

\section{Introduction}

In the early stage of the vehicle industry development, people were all pursuing the extreme driving experience and mechanical property of vehicles. And there is no doubt that the traditional vehicles' mechanical property and dynamic driving performances have reached a very high level, but also entered a plateau period blocking the further evolution of traditional vehicles. (ㅁ) But, when it comes to the early $21^{\text {th }}$ Century, the rise of artificial intelligent has given a new developing direction for the traditional vehicles industry. In addition, the intelligent vehicles are solving the problems which are occurred by the traditional vehicles, such as the driving safety, fatigue driving, and also can set people free from the heavy driving burden.

Every day, almost 3,700 people are killed globally in crashes involving cars, buses, motorcycles or pedestrians. More than half of those killed are pedestrians, motorcyclists, or cyclists. Crash injuries are estimated to be the eighth leading cause of death globally for all age groups and the first leading cause of death for children and young people. As reported by the U.S. Department of Transportation, more than $90 \%$ of car crashes are attributed to drivers' errors. ${ }^{(3)}$ Intelligent vehicles equipped with cameras or Lidar sensors could possess environment perception abilities, which could make self-driving safe and also have artificial intelligence capabilities to assist drivers, like emergency braking. The adoption of intelligent vehicles is expected to improve driving safety and traffic efficiency. Therefore, an accurate environment perception system embedded in intelligent vehicle is an indispensable technique to reduce the traffic accidents.

To maintain an extremely practical self-driving system, the abilities of environment perception like human beings are the indispensable technique. The object detection technology can be divided into two parts from the spatial dimensionality: 2D detection and 3D detection. As is shown in Fig.W, selfdriving system need to detect the objects' position and pose in real 3D spatial application environments. While nowadays, some so-called 3D objects detectors based on 2D detection algorithms achieved very outstanding accuracy and with a great real-time performance. ${ }^{(4)(5)}$ These approaches we mentioned above just rotate the images' angle and transform the $2 \mathrm{D}$ based bounding boxes into $3 \mathrm{D}$ based in foreground of the images. The most disadvantage is the lack of height and $z$ axis information, these detectors neglect the importance of vehicles height information and set the height and $z$ axis information with a universal value by manual. Even though, the width and length of a vehicle is much more important than 
the height information while driving on the road. But the inaccuracy of the height could also leave plenty of bugginess in the darker corners of the implementation. So this paper uses the relationship of height and $\mathrm{z}$ axis value, and predicts the accurate height size of intelligent vehicles from the regressed $z$ axis value. This principle does not always exist, but we still have the second method to predict height value. While vehicles are driving on a bridge or other places not on one horizon line, height size could still be set on a universal value by the vehicle category. And if the height size is in normal vehicles' height range, the first method will be more accurate definitely.

This paper is structured as follows. Section 2 surveys the development process of the 3D detectors for self-driving, and concludes the contributions of this paper. Section 3 describes the whole framework architecture and the original points of this works in details. The accurate and real-time performance of proposed 3D object detection algorithms are evaluated in Section 4. Section 5 provides a brief conclusion of proposed 3D object detection work.

\section{Related Work}

\subsection{D detectors based on 2D detecting networks}

$3 \mathrm{D}$ object detection is an essential technique in the field of self-driving. 3D object detection methods could be divided into 3 categories: vision based, point clouds based and multi-sensor fusion based methods. ${ }^{(6)}$ While currently the point clouds based methods achieves the state-of-the-art performance in 3D detection methods. Due to the accurate depth measurement of point clouds dataset, many frameworks, which used 3D based methods to regress 3D bounding boxes, will bring a lot of computation burdens. While the shortcoming of great amount of computations becomes a challenge to real-time self-driving applications. So many researchers tend to use the $2 \mathrm{D}$ based detection algorithms to realize the object detection in 3 dimensions.

Chen et al. (II) proposed a two-stage network of 3D detection called MV3D in 2017. MV3D transformed the 3D point clouds into BEV (Bird's Eye View) images and FV (front view) images, and fused the normal RGB images, did a mutil-sensor fusion 3D detector. The main idea of MV3D is also to transform the $3 \mathrm{D}$ point clouds data into $2 \mathrm{D}$ image shapes, and then use the $2 \mathrm{D}$ detectors to complete the 3D object detection. Martin et al. ${ }^{(\overline{8})}$ used the YOLOv2 detection network to realized a 3D object detection framework in 2018, and achieved an excellent detection speed to meet the real-time 3D detection in self-driving. In 2018, Shi et $a l{ }^{(\bar{Q})}$ proposed a 3D detector called PointRCNN, which is a two-stage 3D point cloud target detection framework. By dividing the point cloud of the whole scene into foreground and background, the stage-one network directly generates a small number of high-quality $3 \mathrm{D}$ proposals from the point cloud. The stage-two network refines the proposals in the canonical coordinate by combining semantic features and local spatial features. PointRCNN actually used PointNet++(III) to complete a 3D convolution, which has an great improvement in accuracy, but the efficiency is not mentioned. In 2019, Yang et al. (III) present a new two-stage 3D object detection framework, named sparse-to-dense 3D Object Detector (STD). This two-stage network used PointNet++ network as the backbone for $3 \mathrm{D}$ detection. Novelties are a new spherical anchor and $3 \mathrm{D}$ intersection-over-union (IoU), which achieves a high recall and the localization accuracy. This method outperforms other state-of-the-arts, especially on the hard dataset, but with inference speed only around 10 FPS.

According to the related works above, the main contradiction is the balance between detecting accuracy and efficiency. The 3D convolution network based frameworks could obtain high-accurate detection results but the detecting speed is quite slow. In reverse, the 2D detectors based methods, like Complex-YOLO, achieve the high real-time performances, but the detecting accuracy can not be in the front rank of KITTI benchmark. A suitable solution to this problem has been proposed in this paper, and will be explained in details in following text.

\subsection{Contributions}

The previous 2D neural network based 3D detectors like Complex-YOLOv2, Complex-YOLOv3, actually did the 2D object detection, and transformed $2 \mathrm{D}$ bounding boxes into $3 \mathrm{D}$, which is not a real 3D object detection. A real 3D object detection framework should detect the 3D axis value: $(x, y, z)$, and the 3D size of 3D bounding boxes: $(h, w, l)$, finally the rotation angle: yaw. While, this paper have tried the $3 \mathrm{D}$ detectors of 7 DoF (degrees of freedom), we found that the $7 \mathrm{DoF}$ detector has a quite low detection accurate and also can not deal with the real-time driving situations. So, this paper proposed a real $3 \mathrm{D}$ object detector with $6 \mathrm{DoF}$, and could predict all the necessary variables of a 3D object. The contributions of the proposed paper is concluded as below.

(a) The design of 6 DoF neural network

In the modern city, vehicles are always driving on the smooth roads. Based on the this principle, the variable $\mathrm{z}$ of the center point of objects has a transform rela- 


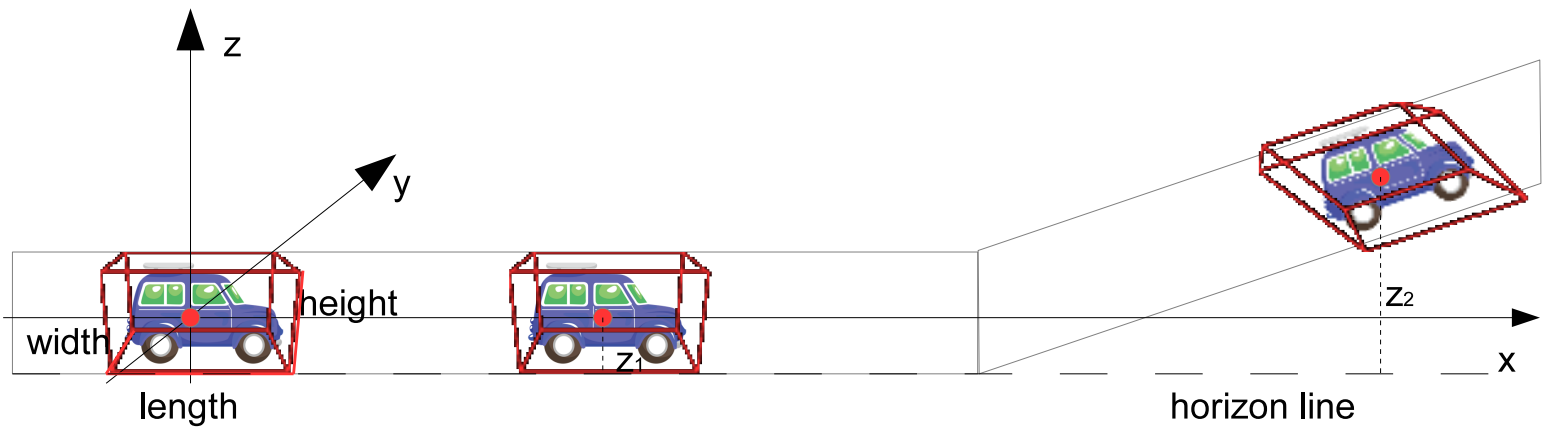

Fig. 1: The metric detection of intelligent vehicles

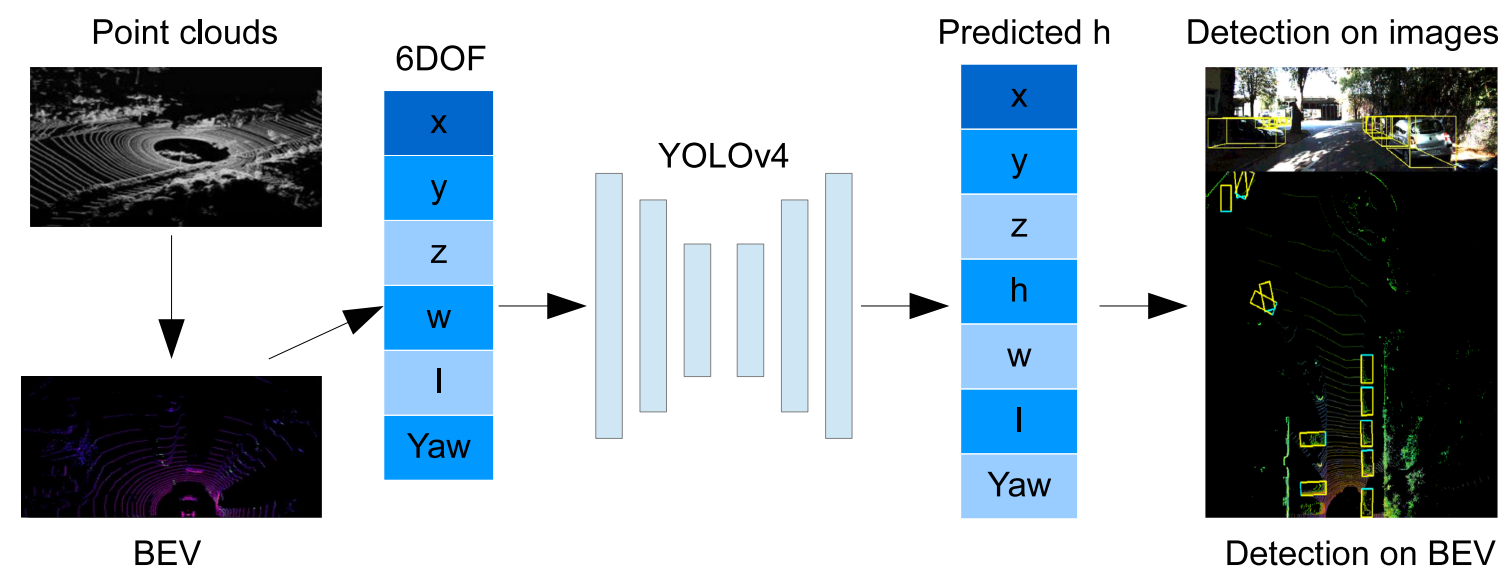

Fig. 2: The architecture of proposed 3D object detection framework

tionship with the height value of object size. So this paper removed the height value which could bring a lot of computation, because the YOLOv4 detection network could use 2D anchor boxes instead of 3D anchor boxes to regress offset values.

(b) The 3D object detection design of YOLOv4 network This paper used the optimized 2D Scaled-YOLOv4 neural network, and transformed it to a 3D detection neural network.

(c) The application of real-time detection

This paper completed a real-time 3D object detector, and could detect the video data of vehicles' front side and back side, which could basically maintain the selfdriving demand at around 27.6 FPS.

\section{The proposed work}

The proposed 3D object detection framework transformed the 3D point clouds dataset into 2D BEV images, and used the optimized complex YOLOv4 network to make the detection work. This paper analyses the shortcomings of the frameworks which used $5 \mathrm{DoF}$ and $7 \mathrm{DoF}$ to regress the final
3D bounding boxes. So this paper proposed the 3D detection frameworks based on $6 \mathrm{DoF}$, which could have a great balance between the accuracy and efficiency in the 3D object detection work, as is shown in Fig.】.

\subsection{The whole architecture of the proposed work}

Due to the rapid development of the $2 \mathrm{D}$ object detection and the outstanding performance on the detecting accuracy and efficiency. This paper also used a 2D detection neural network: YOLOv4, because the 3D object detection with the $3 \mathrm{D}$ convolution network will produce a lot of computation burden, and could not maintain the real time performance of the self-driving.

As the Fig. $\square$ shows, the whole architecture of the proposed $3 \mathrm{D}$ object detection framework is shown as below. The raw point clouds dataset from KITTI Velodyne Lidar is used as the only input data of this framework. The 3D point clouds dataset need to be transformed as BEV images. And the data structures of points clouds dataset are transformed as the traditional RGB images, which could cheat the YOLOv4 framework to input the 2D-image-shape data as usual. Then the optimized YOLOv4 network for 3D detection will extract the features, and do regression and classification works to 3D 
objects in BEV images. Finally the detected 3D coordinate point could be used to make 3D bounding boxes on BEV and detected monocular images for visualization.

\subsection{The preprocessing of KITTI dataset}

With the advent of the YOLOv4 detection network, the state-of-the-art performance and the balance of accuracy and efficiency has been achieved by this detector. This paper used the improved Scaled-YOLOv4 network. But YOLOv4 network only used 2D RGB images as input data. So we need to transform the data structure of point clouds data into 2D image shape with 3 channels. As is shown in Fig.3, we try to add all the detected messages acquired by Velodyne laser scanner into a 3 channels 2D image data structure. From the point clouds dataset, we can get the $(x, y, z)$ of $3 \mathrm{D}$ points and the intensity value of the laser received. We use these messages to calculate and get 3 metric quantities: the density value, the height value and the intensity value. We can use these values as the 3 channels of a 2D image, and then rearrange the 3 channel values according to the coordinate points of $(x, y)$ in a $2 \mathrm{D}$ coordinate system. Thus, we can get a $2 \mathrm{D} \mathrm{BEV}$ images with 3 channels.

\subsection{The design of the training network}

A 3D detected bounding box should have 9 DoF: 3 variables of a centering point, 3 metric variables of a 3D box size, and the 3 variables of rotation yaws. While constricting a real 3D object detection framework on self-driving, the necessary variables could reduced to $7 \mathrm{DoF}$ without the 2 redundant directions of rotation. While in this paper, we are consulting to decrease one more variables to reduce the computation burden while 3D detection. We select the height variable of the 3D boxes, because of the anchor boxes of YOLOv4. The abandon of the height value could greatly decrease the computation amount, because the anchor boxes can be transformed from $3 \mathrm{D}$ to $2 \mathrm{D}$. Besides, the height value could be get from the $\mathrm{z}$ axis of the centering point accurately on a smooth road, which occupied the more than 90 percent of the driving process. The regression of 6 DoF 3D bounding boxes is shown in Fig. 团.

In the regression progress of YOLOv4, the BEV images will be devided into many different grids. If the center points coordinates is detected in some one gird, thus this grid will be responsible for the $6 \mathrm{DoF}$ parameters of the 3D bounding boxes regression. As the Eq.II- Eq.D shows, among the 3D center point coordinate $\left(b_{x}, b_{y}, b_{z}\right)$, the offset $b_{x}$ and $b_{y}$ need to be transformed as the actual coordinate value. The $b_{z}$ value

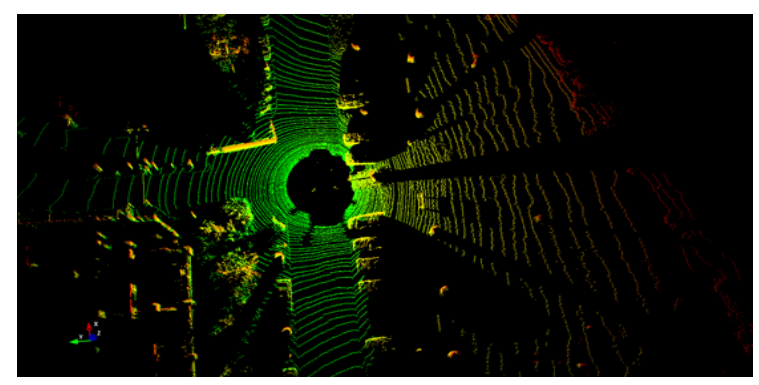

Point clouds variables
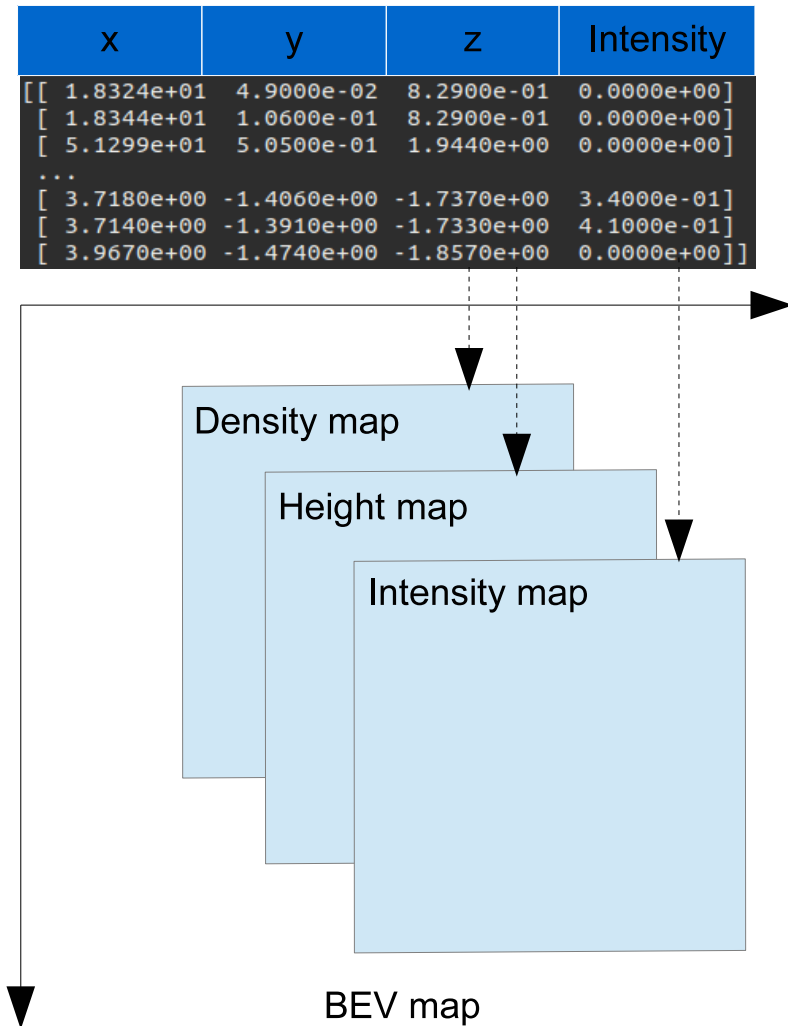

Fig. 3: The preprocessing of point cloud data

is directly regressed because there is only one grid in the vertical axis. Each gird has 5 anchor boxes, and the real value of weight and height of objects can be calculated according to the offset value of $b_{w}$ and $b_{l}$. The height value can be obtained from the relationship of $b_{z}$ and $b_{h}: b_{h}=\left(b_{z}-(-1.73)\right) * 2$. The rotation angle $b_{\varphi}$ is represented in a polar coordinate system, and the rotation angle can be calculated by Eq.6.

$$
\begin{gathered}
b_{x}=\sigma\left(t_{x}\right)+c_{x} \\
b_{y}=\sigma\left(t_{y}\right)+c_{y} \\
b_{z}=\sigma\left(t_{z}\right) \\
b_{w}=P_{w} * e^{t_{w}}
\end{gathered}
$$




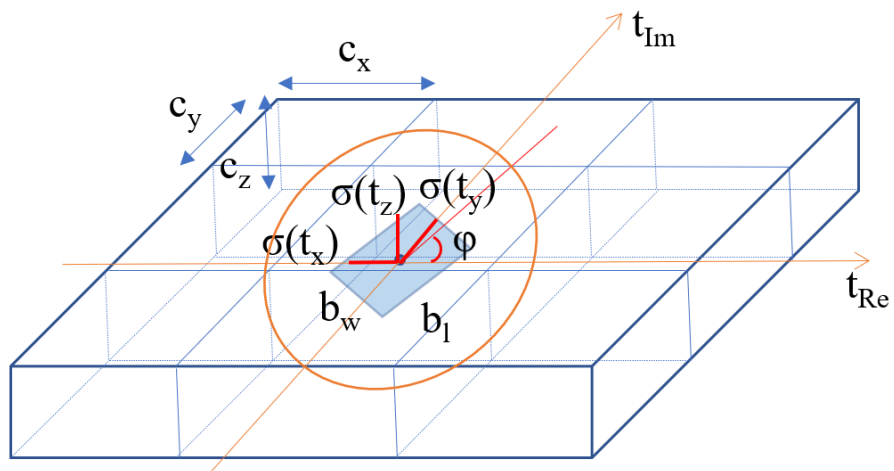

Fig. 4: The regression of $6 \mathrm{DoF}$

$$
\begin{gathered}
b_{l}=P_{l} * e^{t_{l}} \\
b_{\varphi}=\arctan _{2}\left(t_{I m}, t_{R e}\right)
\end{gathered}
$$

\subsection{Loss function}

The proposed 3D detection neural network is based on YOLOv4, so the loss function of the proposed work inspired by YOLOv4 can be devided into 4 part, and the formula is shown in Eq. [- Eq.Ш]. In this formula loss $_{\text {box }}$ represents the size regression of 3D bounding boxes, loss $s_{o b j}$ represents the confidence after IOU, $\operatorname{loss}_{c l s}$ represents the classification loss function, and loss rot represents the rotation angle regression. The design of loss functions is inspired from the YOLOv3, and mainly used the algorithm of MSE (Mean Square Error).

$$
\text { loss }=\operatorname{loss}_{b o x}+\operatorname{loss}_{\text {obj }}+\operatorname{loss}_{c l s}+\operatorname{loss}_{\text {rot }}
$$

$$
\begin{aligned}
\operatorname{loss}_{\text {box }}= & \lambda_{\text {coord }} \sum_{i=0}^{k * k} \sum_{j=0}^{M} I_{i j}^{o b j}\left(2-w_{i} * l_{i}\right)\left[\left(x_{i}-\hat{x}_{i}\right)^{2}\right. \\
& \left.+\left(y_{i}-\hat{y}_{i}\right)^{2}+\left(z_{i}-\hat{z}_{i}\right)^{2}\right] \\
& +\lambda_{\text {coord }} \sum_{i=0}^{k * k} \sum_{j=0}^{M} I_{i j}^{o b j}\left(2-w_{i} * l_{i}\right)\left[\left(w_{i}-\hat{w}_{i}\right)^{2}\right. \\
& \left.+\left(l_{i}-\hat{l}_{i}\right)^{2}\right] \\
\left.\operatorname{loss}_{\text {obj }}\right]= & \sum_{i=0}^{k * k} \sum_{j=0}^{M} I_{i j}^{o b j}\left[\hat{c}_{i} \log \left(c_{i}\right)+\left(1-c_{i}\right) \log \left(1-c_{i}\right)\right] \\
& -\lambda_{\text {noobj }} \sum_{i=0}^{k * k} \sum_{j=0}^{M} I_{i j}^{o b j}\left[\hat{c}_{i} \log \left(c_{i}\right)+\left(1-c_{i}\right) \log \left(1-c_{i}\right)\right]
\end{aligned}
$$

$$
\begin{aligned}
& \operatorname{loss}_{c l s}=-\sum_{i=0}^{k * k} I_{i j}^{o b j} \sum_{c \subset \text { class }}\left[\hat{p}_{i}(c) \log \left(p_{i}(c)\right)\right. \\
&\left.+\left(1-p_{i}(c)\right) \log \left(1-p_{i}(c)\right)\right] \\
& \text { loss }_{\text {rot }}=\lambda_{\text {coord }} \sum_{i=0}^{k * k} \sum_{j=0}^{M} I_{i j}^{o b j}\left[\left(t_{\text {Im }}-t_{\text {Im }}\right)^{2}+\left(t_{R e}-t_{\hat{R e}}\right)^{2}\right]
\end{aligned}
$$

\section{Training and Evaluation}

We trained and evaluated the proposed 6 DoF based 3D detection framework on the point clouds KITTI dataset. This public dataset provides 7,481 samples for training including annotated ground truth and 7,518 test samples with point clouds taken from a Velodyne laser scanner, where annotation data is private. Though, this framework is used to make 3D detection work just as complex-yolo, complex-yolov3. But the traditional Complex-YOLO detection framework used the $2 \mathrm{D}$ evaluate method, while the proposed framework in this paper used the 3D bounding boxes evaluation methods. So the detection accuracy of this work can not be simply compared with the above mentioned complex-YOLO frameworks. The first thing to note is that all our works are all trained and evaluated on a GeForce RTX 2060 Super Graphics cards.

\subsection{The accuracy evaluation of 3D object detection}

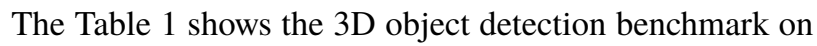
KITTI test set. Though the proposed 3D detection framework did not regress the height value directly, but we can still predict the height value through the value of axis $\mathrm{z}$. In the field of 3D object detection, the proposed work used the 3D evaluation methods, which comparing the detected 3D bounding boxes and the target $3 \mathrm{D}$ bounding boxes. The calculation of IoU also used 3D methods, which uses the 3D volume of two boxes to calculate the CIoU. After the 3D bounding boxes evaluation, we can see that the proposed detector could achieve the $65.86 \%$ in average precision (AP) at 27.6 FPS. The experiment results shows that our work have an extreme performance on 3D object detection.

\subsection{Real-time detections}

This paper finally test the proposed 3D detector in real time video datasets, so that we can test the real-time performance of this detector applied in the real traffic situations. Test video data also used the KITTI dataset "2011-09-26-drive-0014", which contains 320 image frames and 313 velodyne-points 
Table 1: 3D object detection benchmark on KITTI test set

\begin{tabular}{|c|c|c|c|c|c|c|}
\hline Method & Modality & FPS & Car & Pedestrain & Cyclist & AP(IoU = 0.7) \\
\hline MV3D & Lidar+Mono & 2.8 & 62.35 & - & - & - \\
\hline F-PointNet & Lidar+Mono & 5.9 & 70.39 & 44.89 & 56.77 & 57.35 \\
\hline AVOD & Lidar+Mono & 12.5 & 65.78 & 31.51 & 44.90 & 47.39 \\
\hline AVOD-FPN & Lidar+Mono & 10.0 & 71.88 & 39.00 & 46.12 & 52.33 \\
\hline VoxelNet & Lidar & 4.3 & 65.11 & 33.69 & 48.36 & 49.05 \\
\hline Complex-yolo & Lidar & 50.4 & 64.00 & 39.70 & 58.32 & 54.00 \\
\hline PointRCNN & Lidar & - & 75.76 & 41.78 & 59.60 & 59.04 \\
\hline STD & Lidar & - & - & - & - & 62.53 \\
\hline Ours & Lidar & 27.6 & 92.35 & 33.10 & 72.14 & 65.86 \\
\hline
\end{tabular}

data. As is shown in the Fig.[1, We only still select the Cars, Pedestrains and Cyclists as the detecting objects. The FPS of this work could basicly be maintained around the 27.6 FPS, which is demanded for real-time driving situations. Also, we can noticed the FPS would decrease while there are a amount of detection objects being detected at the same time.

\section{Conclusions}

In this paper we present a high-accurate and real-time 3D object detection framework based on point clouds dataset. We have shown the excellent results in terms of accuracy on the KITTI benchmark suite with an outstanding efficiency of around 27.6 FPS (NVIDIA RTX 2060 Super), which achieved a great balance between 3D detecting accuracy and efficiency. This great performance should be attributed to the proposed $6 \mathrm{DoF}$ design of the 3D detection framework, which could actually predict 7 DoF accurately needed by 3D bounding boxes. The decrease of the height DoF makes YOLOv4 to calculate 2D anchors instead of 3D anchors, reducing a lot of computation quantities. The proposed work is able to detect and classify different objects like cars, pedestrians and cyclists simultaneously in real-time dynamic scenes. So our $3 \mathrm{D}$ detection framework has the capability to be applied in self-driving.

\section{References}

(1) Alexey Bochkovskiy, Chien-Yao Wang and Hong-Yuan Mark Liao : "YOLOv4: Optimal Speed and Accuracy of Object Detection”, Computer Vision and Pattern Recognition, 2020

(2) DianGe Yang, Kun Jiang and Ding Zhao : “Intelligent and connected vehicles: Current status and future perspectives" , Science China Technological Sciences, Vol. 61, No. 10, 2018

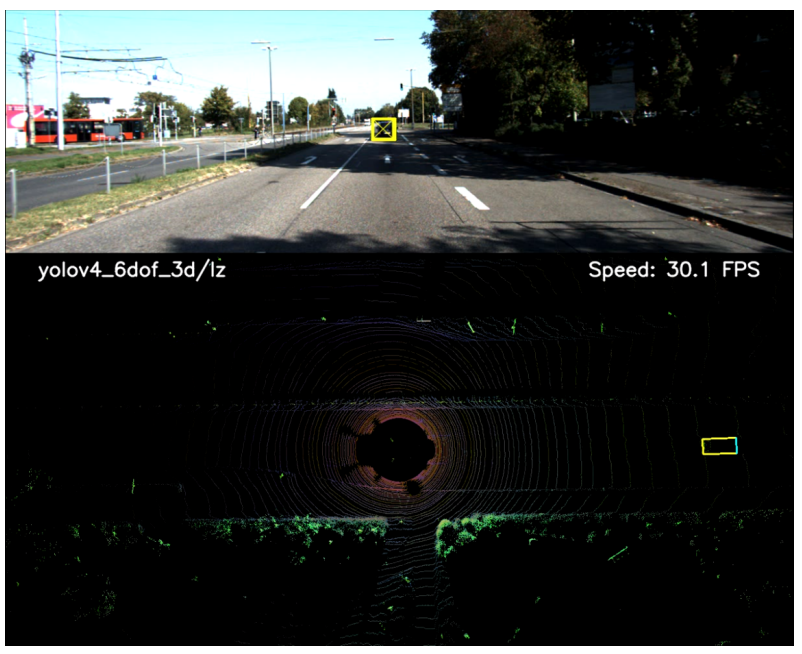

The performance of low-burden transportation framework

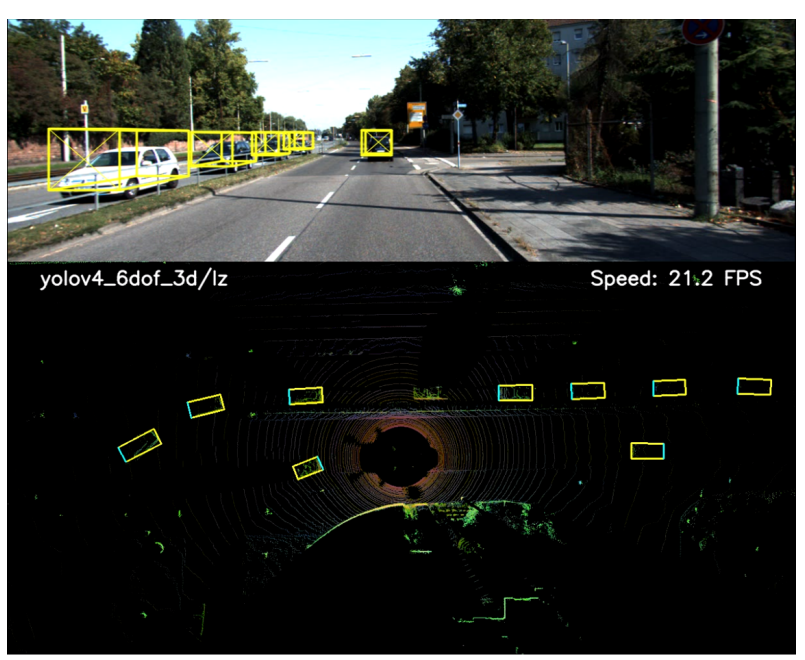

The performance of complex transportation application scence

Fig. 5: The real-time performance of the proposed framework

(3) “Traffic Safety Facts", National Highway Traffic Safety Administration, US Department of Transportation, Tech. Rep. DOT HS 812 115, 2019

(4) Nils Gählert, Junjun Wan and Nicolas Jourdan : “ 
Single-Shot 3D Detection of Vehicles from Monocular RGB Images via Geometry Constrained Keypoints in Real-Time", Computer Vision and Pattern Recognition, 2020

(5) Sourabh Vora, Alex H. Lang and Bassam Helou : "PointPainting: Sequential Fusion for 3D Object Detection ”, IEEE/CVF Conference on Computer Vision and Pattern Recognition, pp.4603-4611, 2020

(6) Zhen Li, Miaomiao Zhu and Shi Zhou : “3D Object Detection Algorithms on Intelligent Driving: A Survey”, Proceedings of The Twenty-Sixth International Symposium on ARTIFICIAL LIFE AND ROBOTICS (AROB 26th 2021) and The Sixth International Symposium on BIOCOMPLEXITY (ISBC 6th 2021), International Society of Artificial Life and Robotics, pp: 38-43, 2021

(7) Xiaozhi Chen, Huimin Ma and Ji Wan : “Multi-view 3D Object Detection Network for Autonomous Driving " , IEEE Conference on Computer Vision and Pattern Recognition (CVPR), pp. 6526-6534, 2017

(8) Martin Simon, Stefan Milz and Karl Amende : "Complex-yolo: Real-time 3d objectdetection on point clouds", Computer Vision and Pattern Recognition, 2018

(9) Shaoshuai Shi, Xiaogang Wang and Hongsheng Li : “PointRCNN: 3D Object Proposal Generation and Detection From Point Cloud”, 2019 IEEE/CVF Conference on Computer Vision and Pattern Recognition (CVPR), pp. 770-779, 2019

(10) Charles R. Qi, Li Yi and Hao Su : “PointNet++: Deep Hierarchical Feature Learning on Point Sets in a Metric Space " Computer Vision and Pattern Recognition, 2020

(11) Zetong Yang, Yanan Sun and Liu Shu : "Std: Sparse-todense 3d object detector for point cloud" , Proceedings of the IEEE/CVF International Conference on Computer Vision, pp. 1951-1960, 2019 